\title{
La révision tarifaire doit rester l'affaire des partenaires tarifaires
}

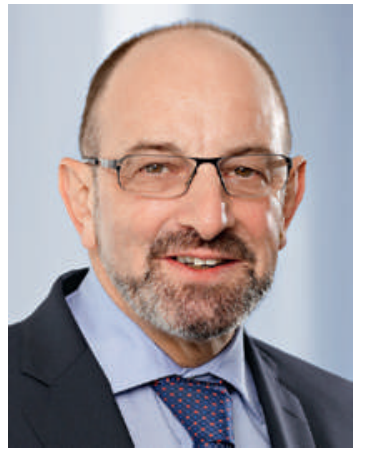

La Commission de la sécurité sociale et de la santé publique du Conseil des Etats (CSSSCE) a déposé une motion intitulée «Tarifs à la prestation distincts dans la LAMal: le Conseil fédéral est chargé, en vue de la future révision de la loi relative à l'admission des médecins dans l'assurance maladie obligatoire, d'examiner notamment une solution prévoyant que la Confédération fixe, en collaboration avec les cantons, des tarifs à la prestation. Ceux-ci seront établis de manière à distinguer les tarifs applicables aux prestations fournies par les spécialistes des tarifs applicables aux prestations fournies par les médecins de premier recours; une distinction sera également opérée en fonction des régions.»

Déposée dans un délai extrêmement court, cette motion vise à réorganiser les compétences en matière de tarif dans les soins ambulatoires. L'approche proposée s'avère également guidée par une vision à court terme: au lieu d'attribuer les tâches et les obligations conformément aux fonctions clairement définies de la Confédération, des répondants des coûts (par ex. santésuisse, CTM) et des prestataires de soins (par ex.

\section{Le développement du tarif doit rester dans les mains des partenaires tarifaires, la $\mathrm{FMH}, \mathrm{H}+$, la CTM et santésuisse, car ils disposent des compétences requises.}

FMH, H+ et toutes les organisations de médecins), la Commission propose de confier le développement des tarifs à l'Etat, ouvrant ainsi clairement la voie à une étatisation de la médecine.

Comme cela a déjà été exprimé dans le Bulletin des médecins suisses (2013; 94), les partenaires tarifaires, la FMH, H+ et la CTM, souhaitent trouver rapidement une solution commune pour la révision du TARMED. Rappelons que le calendrier de la révision tarifaire est respecté et que les objectifs et la feuille de route sont définis par une déclaration d'intention tripartite. Ajoutons enfin que les connaissances spécifiques des sociétés de discipline sont mises en commun avec celles des autres partenaires avant d'être synthétisées et prises en compte dans la nouvelle structure tarifaire. Grâce aux possibilités méthodologiques dont dispose la FMH, il est d'ores et déjà possible de procéder à des simulations pour visualiser les effets des modifications.

Sur le plan technique, la collaboration entre la FMH et santésuisse progresse bien elle aussi. Dans le cadre de l'organi- sation TARMED Suisse, les quatre partenaires ont clairement exprimé leur volonté de conserver l'autonomie tarifaire pour ainsi éviter une intervention de la Confédération. Les négociations visant à élaborer les conditions-cadres nécessaires à la réorganisation de cette entité sont certes difficiles mais elles se déroulent de manière constructive. Les conventions doivent être approuvées et signées d'ici au 30 juin 2013. Si cela réussit, les conditions requises pour que le Conseil fédéral intervienne conformément à sa compétence subsidiaire ne seront pas réunies. L'objectif principal doit être de faire fructifier les compétences propres des partenaires tarifaires (FMH, H+, CTM et santésuisse) pour que le développement du tarif reste dans leurs mains.

\section{La FMH, H+ et la CTM procèdent en- semble à la révision de la structure tarifaire TARMED et peuvent simuler les effets des modifications.}

Les travaux de la FMH ne se résument pas uniquement à la révision de la structure tarifaire TARMED. Dans le cadre du masterplan, ils servent également à la revalorisation de la médecine de famille et fournissent des informations importantes en vue de l'introduction du chapitre 40 dédié aux médecins de premier recours et d'un financement au fil des étapes de la révision globale. Les solutions seront disponibles très prochainement. En rapport avec la révision de la liste des analyses, la FMH avait présenté en 2012 déjà un modèle de calcul basé sur le laboratoire au cabinet, comme le prévoyait le monitorage de l'Office fédéral de la santé publique. Malheureusement, la Confédération n'a pas encore jugé utile d'en discuter avec la FMH.

Dans l'«Info tarifaire importante» de ce numéro (pages 865-866), vous découvrirez la diversité de nos activités. Des changements et des nouveautés sont à l'ordre du jour dans le domaine des tarifs ambulatoires. Par ailleurs, nous négocions et œuvrons à la recherche de solutions dans les projets suivants: pourparlers avec santésuisse sur les procédures EAE, papier de position sur le tiers garant, position de la FMH concernant les dispositions pour la stérilisation, etc. Nous ciblons notre travail et attachons beaucoup d'importance à trouver les meilleures solutions possible avec nos partenaires tarifaires. Nous garderons notre cap et il semble important de le rappeler à la Confédération et au Parlement.

Dr Ernst Gähler, vice-président de la FMH, responsable du domaine Tarifs et conventions pour la médecine ambulatoire en Suisse 